\title{
Analysis of Lift-Off Effect on Transmitter- Receiver Probe in Eddy Current Testing
}

\author{
Qing Zhang ${ }^{\mathrm{a}}$, Xin $\mathrm{Li}^{\mathrm{a}}$ and Guiyun Tian ${ }^{\mathrm{b}, 1}$ \\ ${ }^{a}$ College of Automation Engineering, Nanjing University of Aeronautics and \\ Astronautics, Nanjing, China \\ ${ }^{\mathrm{b}}$ School of Engineering, Newcastle University, England, UK
}

\begin{abstract}
The Transmitter-Receiver Probe (TR probe), which has improved signalto-noise ratio, deep penetration depth, and directional measurement is used in pulsed eddy current testing (PECT). Whereas, the lift-off effect on the TR probe is one of the main obstacles. The purpose of this paper is to analyze the lift-off effects on the TR probe and presents a feature for lift-off estimation. Firstly, an analytical model of a TR probe is given based on previous studies. Secondly, on the basics of the analytical model, the spectrum of the PECT signal is studied, and the lift-off effects on the TR probe are analyzed. Then, the lift-off effects under different coil distances are studied and a $D$ Intersect Point (DIP) is proposed for the lift-off estimation. Results show that the TR probe can be used to reduce the lift-off effect for the nonferromagnetic materials and the ferromagnetic materials. And both the DIP time and amplitude can be used for lift-off estimation. However, the DIP time is better, as its evolution curve with lift-off is a straight line with the same slope. The study is believed to be helpful for the TR probe design, the lift-off measurement, and the thickness assessment and defect quantification.
\end{abstract}

Keywords. Lift-off effect, Transmitter-Receiver Probe, Eddy Current Testing

\section{Introduction}

Different types of Pulsed Eddy Current Testing (PECT) probe have been designed and selected for thickness measurement and defect detection in industrial applications [1]. One of the most commonly used PECT probe configurations includes excitation coil and magnetic sensor probe where a coil is used for excitation and the magnetic sensor is used to pick-up the response signal. Although this type of probe has high sensitivity, only the excitation coil could be optimized [2]. Another type of probe configuration includes two coaxial coils, which use one coil for excitation, and the other for pick-up. Whereas, the Signal-to-Noise Ratio (SNR) of this probe is low, as the signal in the receiver coil is susceptible to the magnetic field directly resulted from the excitation coil [3]. To have directional measurement e.g. stress and improve the SNR, the transmitter and receiver coils are non-coaxial placed, which is called the Transmitter-Receiver probe (TR probe). It has several advantages, such as the improved SNR, the deep penetration depth, etc. [45]. Ona et al. [3] validated that the TR probe sensitivity is enhanced at a higher lift-off when the coil gap exceeds a certain value. These made it a good choice for PECT.

\footnotetext{
${ }^{1}$ Corresponding Author, g.y.tian@newcastle.ac.uk
} 
The lift-off effect of the PECT probe is one of the main obstacles [6]. It is complicated, as it may be affected by many factors, such as specimen materials and probe configurations. That is why the Lift-off Intersection point (LOI) can be easily found in non-ferromagnetic material, while, it is not obvious in ferromagnetic material [7]. Meanwhile, although the lift-off effect has already been studied on the magnetic sensor probe and the coaxial coil probe $[8,9]$, the influence of the lift-off effect on the TR probe has not yet been studied thoroughly [3]. The purpose of this paper is to analyze the liftoff effects on the TR probe and present a feature that can be used for lift-off measurement. Considering a theoretical model serves as a cost-effective and fairly efficient tool to explore the characteristics of the TR probe, the analytical model for the TR probe is used in this paper. Based on the analytical model, the spectrum analysis of PECT signals under different lift-off are given to examine the lift-off effect on the TR probe. Then behaviors of the TR probe under different coil distances are discussed, and a D Intersect Point (DIP) is proposed to measure the lift-off. Moreover, the lift-off effect and DIP are also studied when the specimen is a ferromagnetic plate.

The rest of this paper is organized as follows. In Section 2, the formulation of the TR probe analytical model is given. In Section 3, on the basics of the analytical model, the lift-off effects on the TR probe are analyzed. In Section 4, the lift-off effects are examined under different coil distances $D$, and DIP is proposed to measure the lift-off. In Section 5, the lift-off effect on the TR probe for ferromagnetic material is discussed. And finally, a brief conclusion is given in Section 6.

\section{Formulation of the TR probe analytical model}

Considering the specimen as a metallic plate with the thickness of $c$, and then the TR probe with the lift-off of $l_{0}$ located over the metallic plate is simplified as a three-layered structure shown in Figure 1(a). Layers 1 and 2 represent the air and the metallic plate, respectively. The layer over the plate is considered to be layer 3. It is divided into three sub-regions, and the TR probe which consists of non-coaxial transmitter and receiver coils with the same parameters is located in sub-region II. The probe in polar coordinates is shown in Figure 1(b).
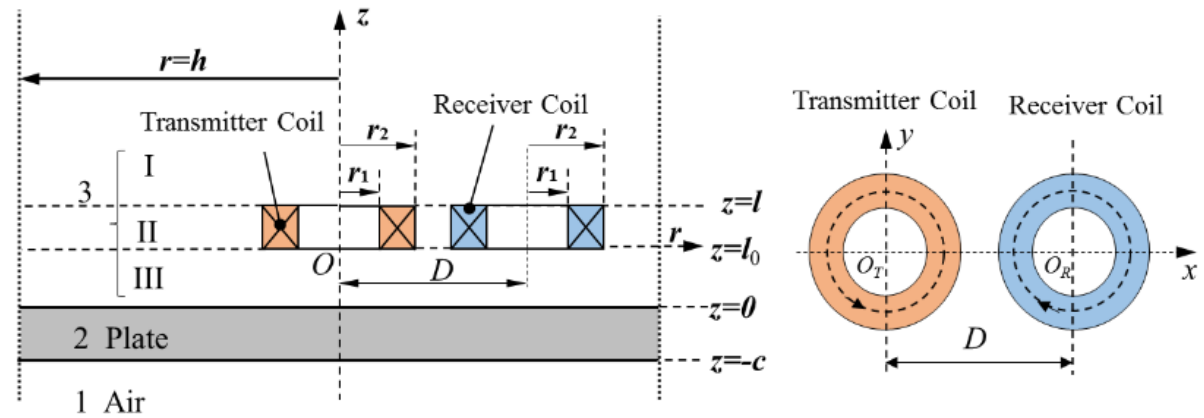

(a) A TR probe over a three-layered structure.

(b) A TR probe in polar coordinates

Figure. 1. The model of the TR probe located over a metallic plate

As the square-wave excitation current of PECT is theoretically represented by superimposing a series of sinusoidal harmonics in the frequency domain, the Fourier- 
based method [10] can be used to obtain the PECT signal $\triangle U$. It can be written as:

$$
\Delta U[s]=\frac{1}{N} \sum_{m=1}^{N} e^{j \frac{2 \pi}{N}(s-1)(m-1)} \Delta U\left[\omega_{m}\right], \quad s=1,2, \ldots, N
$$

where $N$ is the number of sampling points, $m$ denotes the $m$-th sinusoidal harmonic, $\omega_{m}$ is the angular frequency of the $m$-th sinusoidal harmonic, $\triangle U\left[\omega_{m}\right]$ is the induced voltage of the TR probe for the $m$-th sinusoidal harmonic. According to [5], $\Delta U\left[\omega_{m}\right]$ can be expressed as:

$$
\Delta U\left[\omega_{m}\right]=j \pi \omega_{m} \mu_{0} I\left(\omega_{m}\right) \times \int_{0}^{\infty} e^{-2 \alpha l_{0}} \times S(\alpha) \times R_{3,2}^{\prime}(\alpha) \mathrm{d} \alpha
$$

where, $j$ is the imaginary unit, $\mu_{0}$ is the permeability of vacuum, $I\left(\omega_{m}\right)$ is the amplitude of the harmonic excitation current. $l_{0}$ denotes the lift-off of the probe, $\alpha$ can be understood as the radial spatial frequency [11], $S(\alpha)$ is the spatial frequency spectra of the TR probe, and $R_{3,2}^{\prime}(\alpha)$ is the generalized reflection coefficient [12] of the three-layered structure. It can be written as:

$$
R_{3,2}^{\prime}(\alpha)=\frac{\left(\mu_{r 2} \alpha-\beta_{2}\right)\left(\mu_{r 2} \alpha+\beta_{2}\right)-\left(\mu_{r 2} \alpha-\beta_{2}\right)\left(\mu_{r 2} \alpha+\beta_{2}\right) e^{-2 \beta_{2} c}}{\left(\mu_{r 2} \alpha+\beta_{2}\right)\left(\mu_{r 2} \alpha+\beta_{2}\right)-\left(\mu_{r 2} \alpha-\beta_{2}\right)\left(\mu_{r 2} \alpha-\beta_{2}\right) e^{-2 \beta_{2} c}}
$$

where, $\beta_{2}=\left(\alpha^{2}+j \omega_{m} \mu_{0} \mu_{r 2} \sigma_{2}\right)^{1 / 2}, \mu_{r 2}$ and $\sigma_{2}$ are the relative magnetic permeability and electrical conductivity of the metallic plate, respectively.

Moreover, according to [5], the expression of $S(\alpha)$ satisfies:

$$
\begin{array}{r}
S(\alpha)=n^{2} J_{0}(\alpha D)\left[\frac{\left(e^{-\alpha l}-1\right)}{\alpha l_{2}} \times \frac{\operatorname{Int}\left(\alpha r_{1}, \alpha r_{2}\right)}{\alpha^{2}\left(r_{2}-r_{1}\right)}\right]^{2} \\
\operatorname{Int}\left(x_{1}, x_{2}\right)=\int_{x_{1}}^{x_{2}} x J_{1}(x) \mathrm{d} x
\end{array}
$$

where $J_{1}(x)$ denotes the first-order Bessel function, $D$ is the center distance between the transmitter coil and the receiver coil, $n$ is the number of the coil turns, $r_{1}, r_{2},\left(l-l_{0}\right)$ are the inner radius, the outer radius and the height of the coil, respectively.

Meanwhile, as $J_{0}(0)=1$, substituting $J_{0}(0)=1$ into (4), the result is the same with $S(\alpha)$ for the coaxial coil probe in [10]. It indicates that the coaxial probe is a specific TR probe with $D$ equals to 0 . As the coaxial probe is widely used in PECT, it is used as a standard to analyze the performance of the TR probe for reducing the lift-off effect in this paper.

\section{Lift-Off effect on the TR probe}

The formulated model in Section 2 allows efficient and cost-effective investigation of the TR probe behaviors. As the formulated mode has been verified in [5], from now on, simulations were carried out to study the lift-off effect on the TR probe. The metallic plate is considered to be a $20-\mathrm{mm}$ thick aluminum plate. The relative magnetic permeability and conductivity of the aluminum plate are set to be 1 and $21.6 \mathrm{MS} / \mathrm{m}$, respectively. The amplitude, duty cycle, and period of the square-wave current are set to be $4 \mathrm{amps}, 50 \%$, and $1 \mathrm{~s}$, respectively. The parameters of the coils are listed in Table 1 , and the coaxial probe used in this section has the same coil parameters with the TR probe. 
Table 1 Parameters of the probe

\begin{tabular}{crrcc}
\hline Parameters & $\begin{array}{c}r_{1} \\
(\mathrm{~mm})\end{array}$ & $\begin{array}{c}r_{2} \\
(\mathrm{~mm})\end{array}$ & $l-l_{0}(\mathrm{~mm})$ & $n$ \\
\hline Value & 3.0 & 5.0 & 5.0 & 1000 \\
\hline
\end{tabular}

As the behavior of the PECT signal can be revealed by analyzing its spectrum, spectrums of PECT signals under different lift-offs are examined. Figure 2(a) and (b) are the spectrums obtained by the coaxial probe and the TR probe, respectively. As shown in Figure 2, the maximum values of spectrums obtained by the coaxial probe under $0 \mathrm{~mm}$ and $10 \mathrm{~mm}$ lift-offs are $7.49 \mathrm{~V}$ and $0.42 \mathrm{~V}$, respectively. Whereas, those for the TR probe under $0 \mathrm{~mm}$ and $10 \mathrm{~mm}$ lift-offs are $0.434 \mathrm{~V}$ and $0.195 \mathrm{~V}$, respectively. The values of the spectrum for the TR probe are much smaller, and they change much slower with the liftoffs. Therefore, it can be inferred that the signal amplitude of the TR sensor is smaller, and it is insensitive to lift-off.
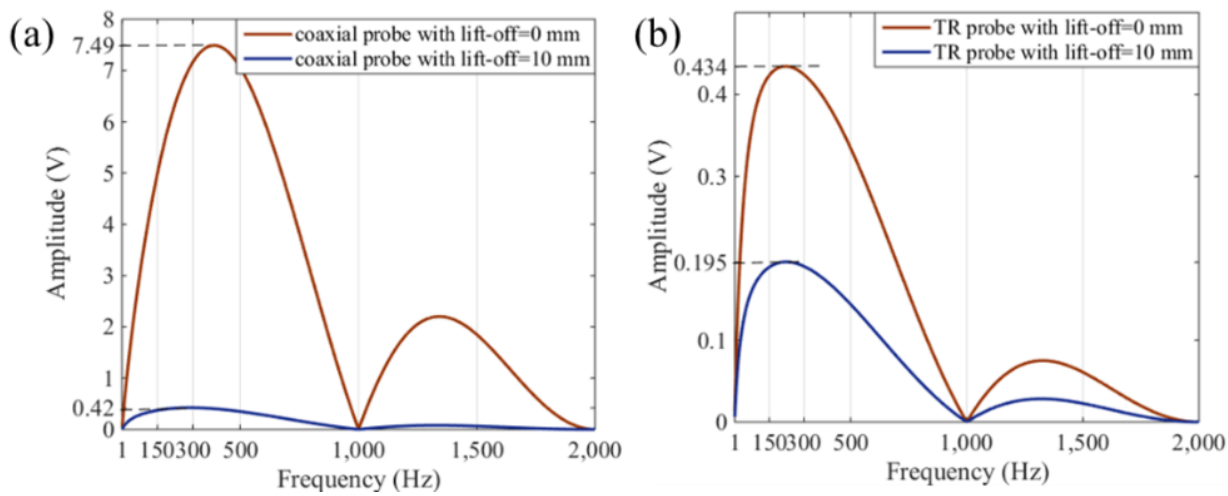

Figure. 2. Spectrum of the PECT signals obtained by (a) the coaxial probe; (b) the TR probe.

(a)

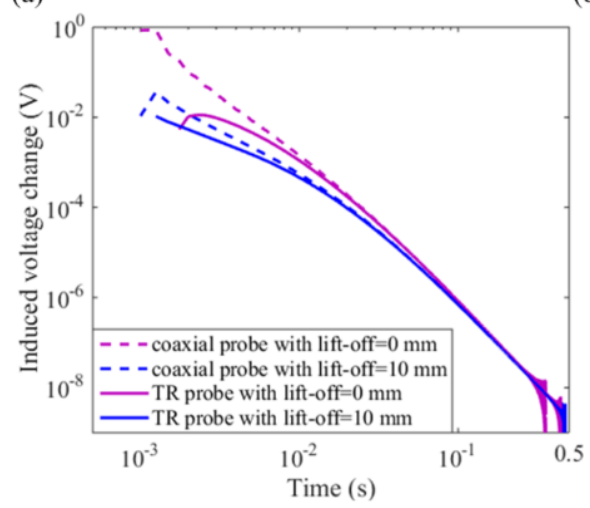

(b)

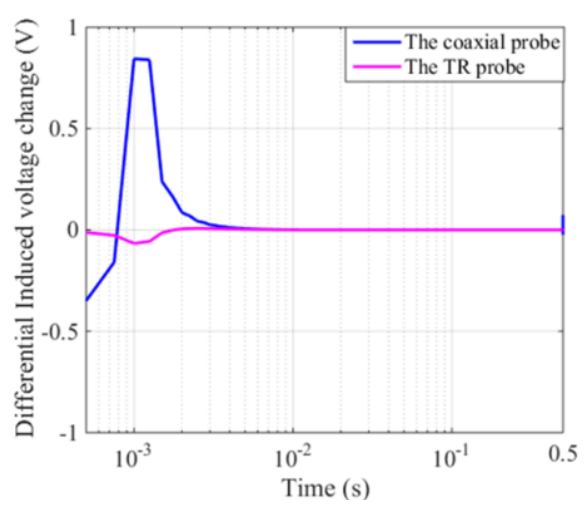

Figure. 3. (a) PECT signals obtained by the coaxial probe and the TR probe under different lift-offs; (b) the differential signals obtained by the coaxial probe and the TR probe.

To verify the above conclusions, signals under $0 \mathrm{~mm}$ and $10 \mathrm{~mm}$ lift-offs are studied. Figure 3(a) are the induced voltage changes $\triangle U$ obtained by the coaxial probe and the TR probe with $D=18 \mathrm{~mm}$. It shows that the signal amplitudes of the TR probe are smaller than those of the coaxial probe, especially in $[0.001,0.010] \mathrm{s}$. Moreover, to investigate the lift-off effect, the corresponding differential signal obtained by subtracting a $10-\mathrm{mm}$ 
lift-off signal from a 0 -mm lift-off signal is given. As shown in Figure 3(b), the difference signal obtained by the TR probe is smaller than that obtained by the coaxial probe. That proves the TR probe can be used to reduce the lift-off effect. These conclusions are consistent with those obtained by the spectral analysis. Furthermore, as shown in Figure 3 (b), the lift-off effect in $[0.01,0.50] \mathrm{s}$ is weak, that is because the maximum spectrum values occur at $150-500 \mathrm{~Hz}$.

\section{Behaviors of the TR probe under different coil distances}

As the coil distance $D$ is an important parameter for the TR probe design, the lift-off effects with different $D$ are discussed in this section. Figure 4(a) are signals of the TR probe with $D=10 \mathrm{~mm}$ and $D=18 \mathrm{~mm}$. It shows that the amplitude of the PECT signal, as well as the difference of $\triangle U$ with $0 \mathrm{~mm}$ and $10 \mathrm{~mm}$ lift-offs, are affected by the coil distance $D$. That indicates the lift-off effect will vary with $D$.

To quantitatively evaluate the lift-off effect, the Euclidean distance is applied. Its calculating formula is:

$$
D i s=\sqrt{\sum\left(\Delta U_{r e f}-\Delta U\right)^{2}}
$$

where $D i$ is is the Euclidean distance, $\triangle U_{\text {ref }}$ is the signal obtained with $0 \mathrm{~mm}$ lift-off, $\Delta U$ is the signal obtained with other lift-offs, and the bigger value of Dis means the more obvious of the lift-off effect.

Through changing the lift-off from 0 to $10 \mathrm{~mm}$ with a step of $2 \mathrm{~mm}$ and $D$ ranging from $10 \mathrm{~mm}$ to $24 \mathrm{~mm}$ with a step of $2 \mathrm{~mm}$, the influence of $D$ on the lift-off effect is discussed. The results are shown in Figure. 4(b), of which $x$-axis denotes the coil center distance and the $y$-axis denotes Euclidean distance Dis obtained by Eq. (6). Figure. 4(b) shows that $D i s$ decreases with $D$ first and then increases, which illustrates that for every lift-off, there exists a specific $D$ which makes the least effective of the lift-off effect. Moreover, for the probe studied in this paper, $D=22 \mathrm{~mm}$ is a good choice. These analyses will be helpful for probe design.

(a)

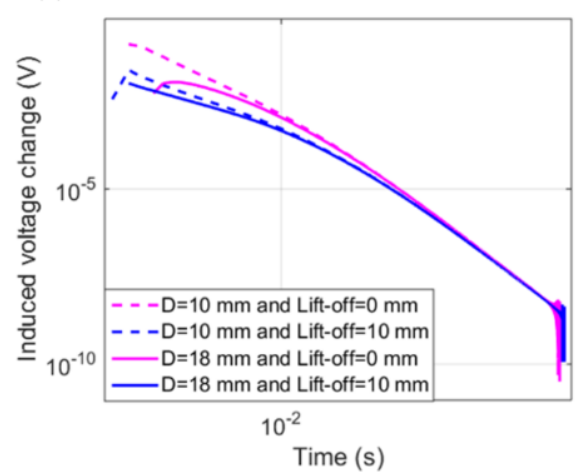

(b)

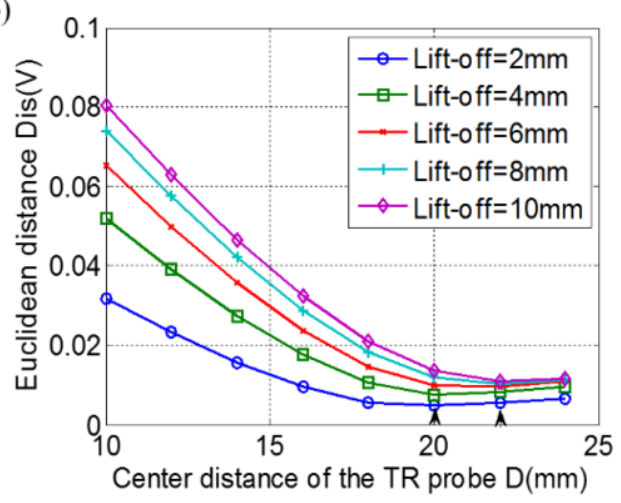

Figure. 4. (a) Signals of the TR probe under different $D$; (b) Euclidean distance of the TR probe under different $D$ and lift-offs.

Furthermore, as differential PECT signals are always used for thickness measurement and defect detection, the differential PECT signals under different coil 
distances are discussed in this section. The differential signal is obtained by subtracting a $16-\mathrm{mm}$ thick aluminum plate signal from a $20-\mathrm{mm}$ thick aluminum plate signal. Figure 5 is the differential PECT signals under $10 \mathrm{~mm}$ lift-off obtained by the TR probe with $D=10 \mathrm{~mm}, 14 \mathrm{~mm}, 18 \mathrm{~mm}$ and $22 \mathrm{~mm}$. The differential PECT signals under different coil distances are intersected at a point, which is called a $D$ Intersect Point (DIP) in this paper.

As the coil distance is related to the lift-off effect on the TR probe, then DIP under different lift-offs are studied. Figure 6(a) shows the behaviors of DIP time with lift-offs and Figure 6(b) shows the behaviors of DIP amplitude with lift-offs. As the lift-off increases, the DIP time and DIP amplitude increases. However, the DIP time evolution curve with lift-off is a straight line with the same slope. This indicates that both the DIP time and DIP amplitude can be used for lift-off measurement, however, the DIP time is better. These are believed to be beneficial for lift-off measurement and high precision detection.

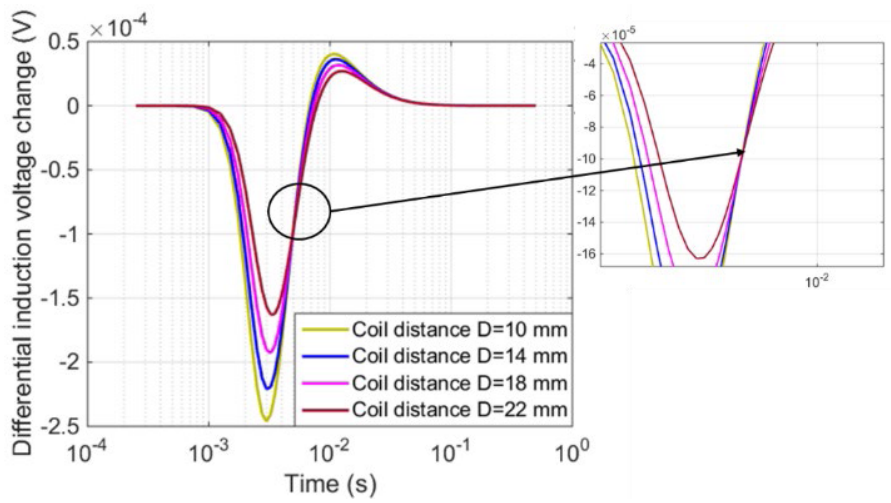

Figure.5. The $D$ Intersect Point under $10 \mathrm{~mm}$ lift-off

(a)

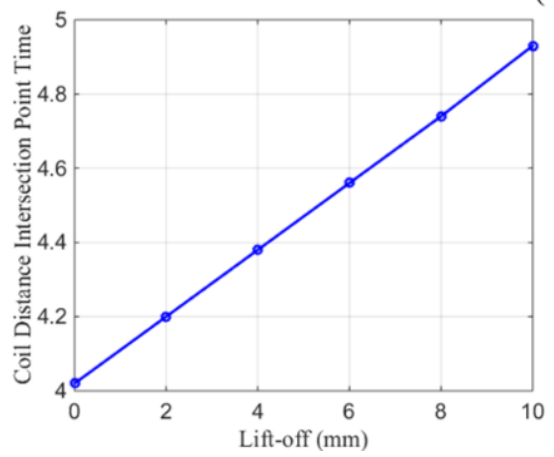

(b)

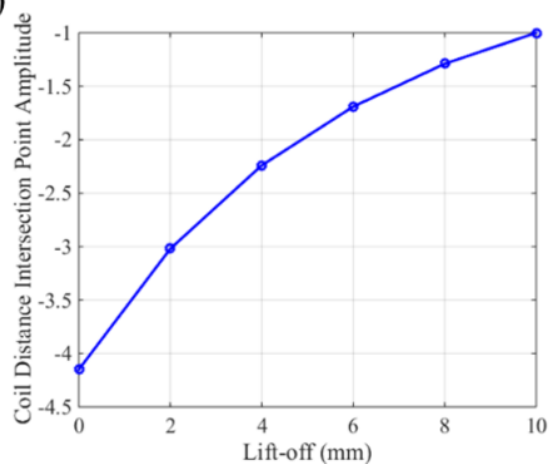

Figure.6. (a) Behavior of the DIP time with lift-offs and (b) behavior of the DIP amplitude with lift-offs

\section{Discussion}

As the specimen could be made by a ferromagnetic material, and the signal feature for a ferromagnetic plate and a non- ferromagnetic plate may be different, the behavior of the TR probe for a ferromagnetic plate is discussed in this section. The ferromagnetic plate 
is represented by a $20-\mathrm{mm}$ thick $\mathrm{U} 71 \mathrm{Mn}(\mathrm{G})$ plate, which is widely used in rail. And the relative magnetic permeability and conductivity of the $\mathrm{U} 71 \mathrm{Mn}(\mathrm{G})$ plate are 1000 and 2.0 $\mathrm{MS} / \mathrm{m}$, respectively.

Figure $7(\mathrm{a})$ and (b) give the induced voltage changes $\triangle U$ for the $\mathrm{U} 71 \mathrm{Mn}(\mathrm{G})$ plate obtained by the coaxial probe and the TR probe with $D=18 \mathrm{~mm}$, respectively. Figure 7(c) and (d) are the corresponding spectrum obtained by the coaxial probe and the TR probe, respectively. Results show that the TR probe can be used to reduce the lift-off effect for the ferromagnetic materials. Moreover, different from the aluminum plate, the lift-off effect of the $\mathrm{U} 71 \mathrm{Mn}(\mathrm{G})$ plate in $[0.001,0.01] \mathrm{s}$ is weak, this is because of the maximum spectrum values for the U71Mn(G) plate occur at $1 \mathrm{~Hz}$. Moreover, as shown in Figure 8, the DIP is also found for the U71Mn $(\mathrm{G})$ plate, and the DIP time evolution curve with lift-off is also a straight line with the same slope. That means the DIP time and amplitude can also be used for lift-off measurement when the specimen is a ferromagnetic plate.

(a)

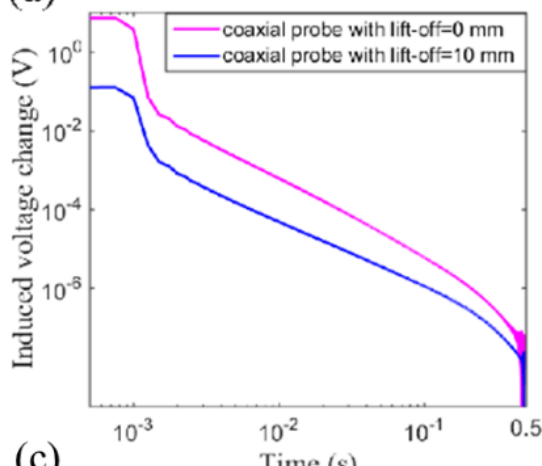

(c)

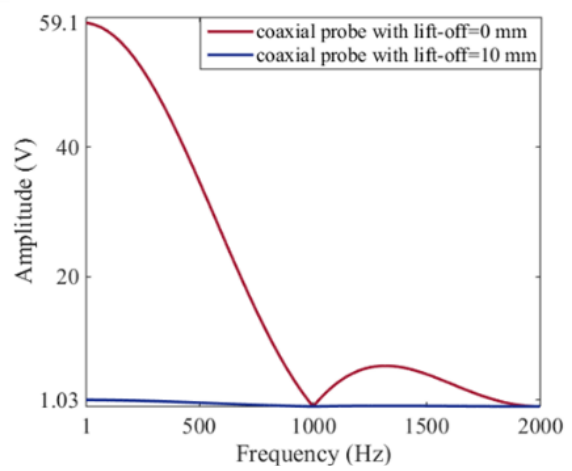

(b)

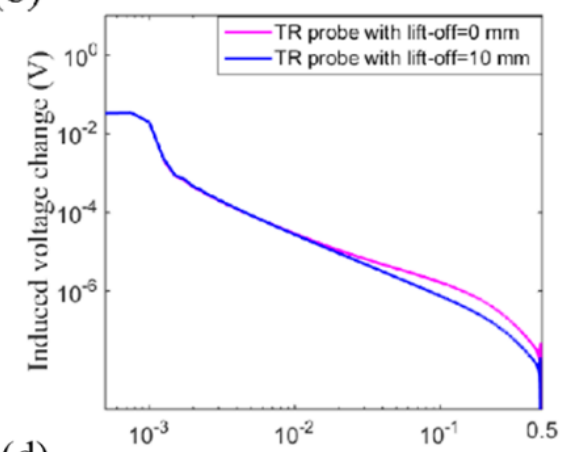

(d)

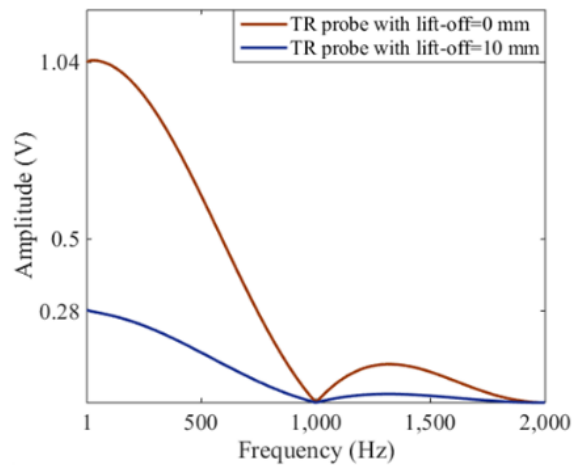

Figure.7. PECT signals obtained by (a) the coaxial probe; (b) the TR probe; and spectrum of the PECT signals obtained by (c) the coaxial probe; (d) the TR probe. 


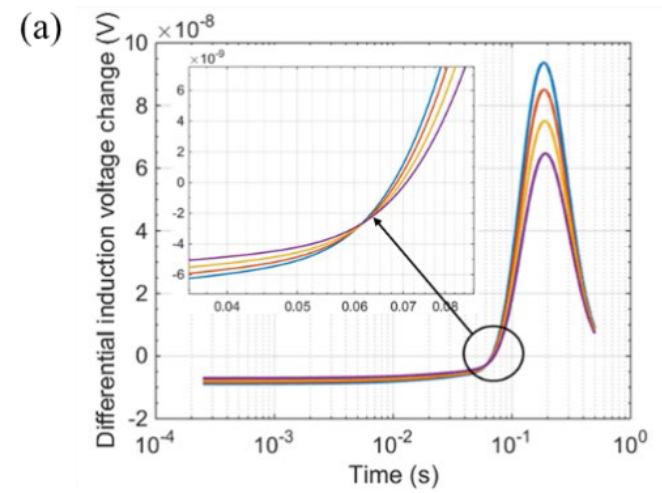

(b)

(c)
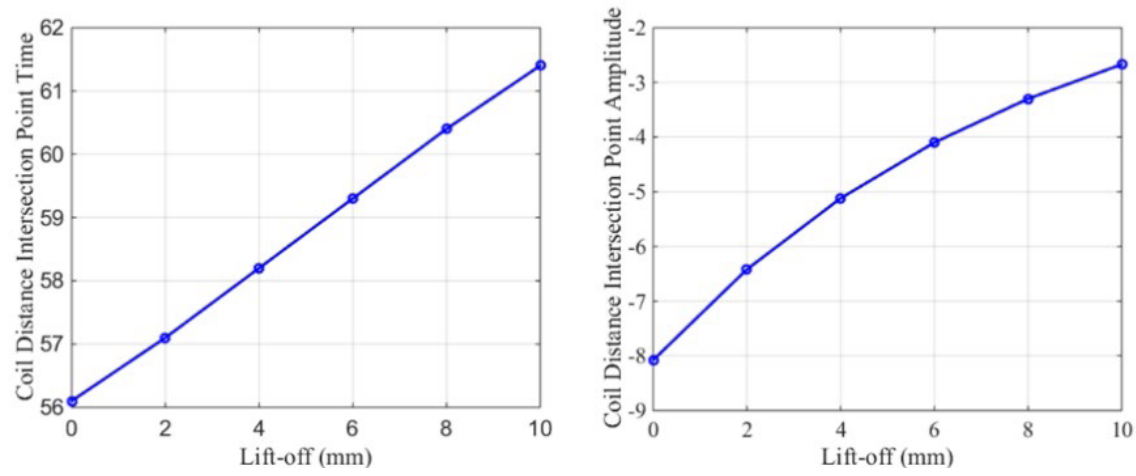

Figure.8. (a) DIP for the U71Mn(G) plate; (b) behavior of the DIP time with lift-offs and (c) behavior of the DIP amplitude with lift-offs

\section{Conclusion}

The lift-off effects and the $D$ Intersect Point (DIP) phenomenon on the TR probe are studied in this paper based on an analytical model. Firstly, by discussing the spectrum and the signals under different lift-offs, it proves that the TR probe can be used to reduce the lift-off effect both for the ferromagnetic and non-ferromagnetic materials. Then, the lift-off effects under different coil distances and the DIP phenomenon are examined. Results show that there exists a specific $D$ which makes the least effective of the lift-off effect. The DIP time and amplitude can be used for lift-off estimation, of which the linearity of DIP time and lift-off is better. These are beneficial for the probe design, the lift-off measurement, and the high precision detection. The further work will focus on the experimental study of the TR probe for the defect detection, speed effect, and the TR probe design and optimization with the variation of lift-offs. 


\section{Acknowledgment}

This work was supported by the National Natural Science Foundation of China under Grant 61903193 and Major Instrument Project of National Science Foundation of China under Grant 61527803.

\section{References}

[1] A. Sophian, G. Tian, and M. Fan, Pulsed eddy current non-destructive testing and evaluation, A review, Chinese Journal of Mechanical Engineering 30.3 (2017), 500-514.

[2] Z. Xu, X. Wang, and Y. Deng, Rotating Focused Field Eddy-Current Sensing for Arbitrary Orientation Defects Detection in Carbon Steel, Sensors 20.8 (2020), 2345.

[3] O. D. Ijike, G. Y. Tian, R. Sutthaweekul, S. M. Naqvi, Design and optimisation of mutual inductance based pulsed eddy current probe, Measurement 144 (2019), 402-409.

[4] G. Mook, O. Hesse, V. Uchanin, Deep penetrating eddy cur-rents and probes, Mater. Test 49 (2007), 258264.

[5] Q. Zhang, and X. Wu, Study on the Shielding Effect of Claddings with Transmitter-Receiver Sensor in Pulsed Eddy Current Testing, Journal of Nondestructive Evaluation 38.4 (2019), 99.

[6] G. Y. Tian and A. Sophian, Reduction of lift-off effects for pulsed eddy current NDT, NDT\&E Int. 38.1 (2005), 319-324.

[7] D. Wen, M. Fan, B Cao, and B. Ye, A Twice Subtraction Method for Obtaining LOI in Pulsed Eddy Current Signals of Ferromagnetic Samples. IEEE Trans. Magn. 55.12 (2019), 1-7.

[8] G. Y. Tian, Y. Li and C. Mandache, Study of lift-off invariance for pulsed eddy-current signals, IEEE Trans. Magn. 45.1 (2009), 184-191.

[9] J. Li, X. Wu, Q. Zhang, and P. Sun, Measurement of lift-off using the relative variation of magnetic flux in pulsed eddy current testing, NDT\&E Int. 75 (2015), 57-64.

[10] Z. Xu, X. Wu, J. Li, and Y. Kang, Assessment of wall thinning in insulated ferromagnetic pipes using the time-to-peak of differential pulsed eddy-current testing signals, NDT\&E Int. 51(2012), 24-29.

[11] V. O. De Haan and P. A. De Jong, Analytical expressions for transient induction voltage in a receiving coil due to a coaxial transmitting coil over a conducting plate, IEEE Trans. Magn. 40.2 (2004), 371-378.

[12] M. Fan, P. Huang, B. Ye, D. Hou, G. Zhang, and Z. Zhou, Analytical modeling for transient probe response in pulsed eddy current testing, NDT\&E Int. 42 (2009), 376-383. 